\title{
A SINGULAR FUNCTIONAL
}

ALLAN D. MARTIN ${ }^{1}$

1. Introduction. This paper is a note to a paper [2] written by the author with Walter Leighton, in which a quadratic functional with a singular end point is studied. The results obtained here extend and amplify those of that paper.

We consider a functional

$$
\left.J(y)\right|_{x} ^{b}=\int_{x}^{b}\left[r y^{\prime 2}+2 q y y^{\prime}+p y^{2}\right] d x \quad(0<x<b),
$$

in which the functions $r, p$ and $q$ are continuous on $(0, \infty)$ while $r$ is positive there. The point $x=0$ is singular for the functional in the sense that the conditions on $r, p$, and $q$ may not hold for an interval of the form $[0, b]$. Finally, all integrals which appear are Lebesgue integrals. We denote by: $F[0, b]$, the class of all functions $y$ such that $y$ is absolutely continuous and $y^{\prime} \in L^{2}$ on every closed subinterval of $(0, b]$ while $y(b)=0 ; F^{\prime}[0, b]$, the class of all functions $y$ such that $y \in F[0, b]$ and $y$ is bounded on $[0, b] ; F_{0}[0, b]$, the class of all $y \in F[0, b]$ for which the point $x=0$ is a limit point of zeros of $y$; $A[0, b]$, the class of all $y \in F[0, b]$ for which $y$ is continuous on $[0, b]$ and for which $y(0)=0 ; A_{0}[0, b)$, the class of all $y \in A[0, b]$ for which the point $x=0$ is a limit point of zeros of $y$. For a function $y$ in any one of the above classes $\left.J(y)\right|_{x} ^{b}$ exists as a Lebesgue integral and is finite for $0<x \leqq b$. We extend its definition by the relation

$$
\left.J(y)\right|_{0} ^{b}=\left.\liminf _{x=0+} J(y)\right|_{x} ^{b} .
$$

(Hereafter, all limits, limits inferior and limits superior will be understood to be taken at $x=0^{+}$unless otherwise noted.) Clearly the value of $J$ may be positively or negatively infinite at $x=0$.

We consider conditions under which $\left.J(y)\right|_{0} ^{b} \geqq 0$ for every $y \in X[0, b]$, where $X[0, b]$ denotes any one of the classes defined above. We shall express this verbally by the statement that $J$ has an $X$-minimum limit on $[0, b]$.

The notions of conjugate and focal points are defined in the usual way. Let $u$ and $v$ be nonnull solutions of the Euler equation, $\left(r y^{\prime}+q y\right)^{\prime}$

Received by the editors June 8, 1955 and, in revised form, November 14, 1955.

1 This research was supported by the United States Air Force through the Office of Scientific Research of the Air Research and Development Command. 
$-\left(q y^{\prime}+p y\right)=0,(0<x \leqq b)$, such that $u(a)=0$ and $r(a) v^{\prime}(a)+q(a) v(a)$ $=0$. Then the conjugate point, $x=c(a)$, of the point $x=a$ is defined to be the first zero of $u$ lying to the right of $x=a$. The focal point, $x=f(a)$, of the line $x=a$ is defined to be the first zero of $v$ which lies to the right of $x=a$. If $u$ or $v$ has no such zero then $x=\infty$ is taken as the corresponding point. The functions $f$ and $c$ are defined finite or infinite for every positive $x$. Both $f$ and $c$ are continuous while $c$ is monotonically increasing. Finally we assign values to $f$ and $c$ at $x=0$ by means of the relations $c(0)=\lim c(x)$ and $f(0)=\lim \inf f(x)$.

2. The $A_{0^{-}}$and the $A$-minimum limit. By a slight modification of the proof of Theorem 7.1 in [2] we have the following result.

THEOREM 2.1. In order that an interval $[0, b]$ afford an $A_{0}$-minimum limit to $J$ it is necessary that $c(0) \geqq b$ and that

$$
\left.J(y)\right|_{0} ^{b}>-\infty
$$

for every $y \in F^{\prime}[0, b]$.

It is relevant to ask to what extent the conditions of the theorem are sufficient. As we shall see, the conditions are not sufficient for an $A$-minimum limit but do suffice to guarantee the existence of an $A_{0}$-minimum limit. In fact we have a stronger result.

THEOREM 2.2. If $c(0) \geqq b$ and if (2.1) holds for every $y \in A_{0}[0, b]$ then $\left.J(y)\right|_{0} ^{b} \geqq 0$ for all $y \in A_{0}[0, b]$.

Suppose on the contrary that $c(0) \geqq b$ and that there exists $y \in A_{0}[0$, $b]$ such that $\left.J(y)\right|_{0} ^{b}<-1$. Let $x_{n}$ and $t_{n}$ be two sequences whose points lie on $(0, b)$ such that $x_{n+1}<t_{n}<x_{n}, y\left(x_{n}\right)=y\left(x_{n+1}\right)=0$ and such that $\left.J(y)\right|_{i_{n}} ^{b}<-1,(n=1,2, \cdots)$. Now since $c(0) \geqq b$ we have by the definition of $x_{n}$ that for all $n,\left.J(y)\right|_{x_{n}} ^{b} \geqq 0$ and thus $\left.J(y)\right|_{t_{n}} ^{x_{n}}<-1$. Thus $y$ is not identically zero in $\left[x_{n+1}, x_{n}\right]$ and if $M_{n}=\max |y(x)|\left(x_{n+1}\right.$ $\left.\leqq x \leqq x_{n}\right)$ then $M_{n}>0$ for every $n$ and $\lim _{n=\infty} M_{n}=0$. We define a function $z \in F^{\prime}[0, b]$ as follows:

$$
z(x)=\left\{\begin{array}{lr}
0 & \left(x_{1} \leqq x \leqq b\right), \\
M_{n}^{-1 / 2} y(x) & \left(x_{n+1} \leqq x \leqq x_{n}\right) .
\end{array}\right.
$$

Now let $M_{m(n)}$ be a subsequence such that $M_{m(n)} \leqq M_{k}$ for all $k<m(n)$. By the definition of $M_{n}$ such a sequence exists. We assert that $\left.\lim _{n=\infty} J(z)\right|_{\imath} ^{b}=-\infty, t=t_{m(n)}$. We then have

$$
\left.J(z)\right|_{t_{m(n)}} ^{b}=\left.\sum_{k=1}^{m(n)-1} M_{k}^{-1} J(y)\right|_{x_{k+1}} ^{x_{k}}+\left.M_{m(n)}^{-1} J(y)\right|_{t_{m(n)}} ^{x_{m}(n)} .
$$


Now since $c(0) \geqq b$ and thus $\left.J(y)\right|_{x_{k+1}} ^{x_{k}} \geqq 0$, we have

$$
\left.J(z)\right|_{i_{m(n)}} ^{b} \leqq\left. M_{m(n)}^{-1} J(y)\right|_{t_{m(n)}} ^{b} \leqq-M_{m(n)}^{-1} .
$$

Thus $\left.\lim _{n-\infty} J(z)\right|_{i_{m(n)}} ^{b}=-\infty$, the assertion follows and the theorem is proved.

The preceding two theorems give the following result.

TheOREm 2.3. If an interval $[0, e]$ affords an $A_{0}$-minimum limit to $J$ then any interval $[0, b]$ for which $c(0) \geqq b$ also affords an $A_{0}$-minimum limit to $J$.

3. The $A$-minimum limit. It was remarked in the previous section that if Theorem 2.2 is stated with $A[0, b]$ replacing $A_{0}[0, b]$, the result is false. A reason for this is contained in the following theorem.

TheOREM 3.1. If $J$ has an A-minimum limit on an interval $[0, e]$ then $J$ has an $A$-minimum limit on any interval $[0, b]$ for which $c(0)$ $\geqq b$ if the Euler equation $\left(r y^{\prime}+q y\right)^{\prime}-\left(q y^{\prime}+p y\right)=0$ has a solution $u$ such that lim sup $|u(x)|>0$. If for every solution $u$ of the Euler equation $\lim \sup |u(x)|=0$, then $[0, b]$ affords an $A$-minimum limit to $J$ if and only if $f(0) \geqq b$.

The first statement of the theorem is contained in the proofs of Corollary 7.1 and Theorems 7.4-7.5 of [2]. As for the second part, it follows by Theorem 8.2 of [2] that if $f(0) \geqq b$ then $[0, b]$ affords an $F$-minimum limit to $J$. This establishes the sufficiency. To verify the necessary half of the result suppose, on the contrary, that $[0, \mathrm{~b}]$ affords an $A$-minimum limit to $J$ but $f(0)<b$. By Theorem 2.1, we may suppose that $c(0) \geqq b$. Let $u$ be a nonnull solution of the Euler equation such that $u(c)=0$ where $f(0)<c<b$. Now if $z$ is a function defined by the conditions that $z(x)=0$ if $c \leqq x \leqq b, z(x)=u(x)$ if $0<x \leqq c$, while $z(0)=0$, then $z \in A[0, b]$. Further, there exists a sequence of numbers $x_{n}$ tending to zero such that $\left.J(u)\right|_{x_{n}} ^{c}<0$. This follows from the fact that $f(0)<c$. Thus $\lim$ inf $\left.J(u)\right|_{x} ^{c} \leqq 0$. Now let $v$ be a nonnull solution such that if $0<x_{1}<c<x_{2}<b, v\left(x_{1}\right)=u\left(x_{1}\right)$ and $v\left(x_{2}\right)=0$. Since $c(0) \geqq b$, the solution $v$ exists and is unique. By Sturm's theory, $\left.J(v)\right|_{x_{1}} ^{x_{2}}<\left.J(u)\right|_{x_{1}} ^{c}$. Now if $y$ is a function for which $y(x)=0$ for $x_{2} \leqq x \leqq b, y(x)=v(x)$ for $x_{1} \leqq x \leqq x_{2}, y(x)=u(x)$ for $0<x \leqq x_{1}$ while $y(0)=0$, then $y \in A[0, b]$. Thus we have the relations

$$
\left.J(y)\right|_{0} ^{b}=\left.J(v)\right|_{x_{1}} ^{x_{2}}+\left.J(u)\right|_{0} ^{x_{1}}<\left.J(u)\right|_{x_{1}} ^{c}+\left.J(u)\right|_{0} ^{x_{1}}=\left.J(u)\right|_{0} ^{c} \leqq 0 .
$$

Thus $J(y)_{0}^{b}<0$ and the proof is complete.

We continue with a sufficiency condition on functionals for which 
the function $q$ vanishes identically. The interest in it lies in the fact that it consists of two necessary conditions and a third condition which is contragradient to the crude notion that for a positive functional $r$ should not be too small and $p$ not too negative.

TheOREM 3.2. If $q=0, c(0) \geqq b$, $\lim$ inf $\int_{x}^{b} p(t) d t>-\infty$ and if $\lim \sup r(x) p(x)<\infty$ then $J$ has an A-minimum limit on $[0, b]$.

By Theorem 7.5 of [2] it is sufficient to show that there exists an interval $[0,3]$ which affords an $A$-minimum limit to $J$. If $u$ is a nonnull solution of the Euler equation it will vanish at most once on $(0, b)$ by virtue of the hypothesis $c(0) \geqq b$. Thus by Theorem 6.2 of [2] we have

$$
\left.J(y)\right|_{x} ^{c}=\int_{x}^{c} r(t)\left[y^{\prime}(t)-\frac{u^{\prime}(t)}{u(t)} y(t)\right]^{2} d t-r(x) \frac{u^{\prime}(x)}{u(x)} y^{2}(x)
$$

$$
(0<x \leqq c)
$$

for every $y \in A[0, c]$, if $u$ is not zero on $(0, c)$. Furthermore it is well known that the function $r(x) u^{\prime}(x) / u(x)$ is a solution of the Riccati equation $z^{\prime}+z^{2} / r-p=0$. By formula (3.1) it follows that the theorem will be proved if we show that $\lim \sup r(x) u^{\prime}(x) / u(x)<\infty$ for at least one nonnull solution of the Euler equation. If there exists a solution of the Riccati equation which is not monotonic near $x=0$ then there exists a sequence $x_{n}$ tending to $0^{+}$on which $r(x) u^{\prime}(x) / u(x)$ attains its limit superior and at each point of which its derivative vanishes. From the Riccati equation it follows that for $x=x_{n}$, $\left(r(x) u^{\prime}(x) / u(x)\right)^{2}=r(x) p(x)$ and the result follows from the hypothesis on $r(x) p(x)$. In the remaining case every solution of the Riccati equation is monotonic and the theorem remains unproved only in the case where every solution of the Riccati equation is monotonically decreasing near $x=0$ and is eventually positive there. If $\int_{0}^{b} r^{-1}(x) d x$ $<\infty$ then since $p(x)=r(x) p(x) / r(x)$ it follows from our hypothesis that $\int_{x}^{b} p(t) d t=O(1)$ as $x$ tends to zero and the result follows from Theorem 9.1 of [2]. Otherwise $\int_{0}^{o} r^{-1}(x) d x=\infty$ and if $r(x) u^{\prime}(x) / u(x)$ is eventually positive for every solution $u$ of the Euler equation it follows that $\lim u(x)$ exists and is finite for every solution $u$. Thus for this case, $\int_{0}\left(r(x) u^{2}(x)\right)^{-1} d x=\infty$ for every non-null solution $u$ and an interval $(0, e]$ on which $u(x)$ is not zero. But this is impossible since by Theorem 5.1 of [2] the Euler equation of a functional for which $c(0)>0$ always possesses a solution $u$ for which $\int_{0}\left(r(x) u^{2}(x)\right)^{-1} d x<\infty$. The theorem is proved.

In the above theorem the conditions $c(0) \geqq b$ and $\lim$ inf $\int_{x}^{b} p(t) d t$ 
$<\infty$ are both necessary conditions. This follows in the latter condition by taking in Theorem 2.1 a function in $F^{\prime}[0, b]$ which is identically equal to the constant one near $x=0$. These two conditions even together are not sufficient for the existence of an $A$-minimum limit in a functional for which $q \equiv 0$ (see [2, Example 9.1]). Therefore the assumptions comprise a sufficient condition because of the presence of an assumption which appears to contribute nothing to the making of a positive functional.

4. The $F_{0}$-minimum limit. We begin with a lemma.

$$
\begin{aligned}
& \text { Lemma 4.1. If } f(0)=0 \text { then there exists } y \in F_{0}[0, b] \text { such that }\left.J(y)\right|_{0} ^{b} \\
& =-\infty \text {. }
\end{aligned}
$$

A proof of this result may obtained by a minor alteration of the last part of the demonstration of Theorem 7.1 in [2].

THEOREM 4.1. The functional $J$ has an $F_{0}$-minimum limit on $[0, b]$ if and only if $c(0) \geqq b$ and $f(0)>0$.

The necessity of the conditions is an immediate consequence of Theorem 2.1 and the above lemma. Conversely let $y \in F_{0}[0, b]$ and let $e$ and $d$ be numbers such that $0<d<e<f(0)$ and such that if $0<a<d$ the focal point of the line $x=a$ lies on $(e, \infty)$. The hypothesis assures the existence of these numbers. Let $x=x_{0}$ be a zero of $y$ which lies in $(0, d)$. Then $\left.J(y)\right|_{x_{0}} ^{b} \geqq 0$. Moreover since $f(x)>x_{0}$ when, $0<x<x_{0}$ it follows that $\left.J(y)\right|_{x} ^{x_{0}} \geqq 0$ for $0<x<x_{0}$. The theorem is proved.

We recall that the conditions $c(0) \geqq b$ and $f(0) \geqq b$ impart to $J$ an $F$-minimum limit on $[0, b]$. (See Theorems 8.1 and 8.2 of [2]). It is also clear that Theorem 2.2 holds if $A_{0}$-minimum limit is replaced by $F_{0}$-minimum limit.

\section{BIBLIOGRAPHY}

1. Walter Leighton, Principal quadralic functionals, Trans. Amer. Math. Soc. vol. 67 (1949) pp. 253-274.

2. Walter Leighton and Allan D. Martin, Quadratic functionals with a singular endpoint, Trans. Amer. Math. Soc. vol. 78 (1955) pp. 98-128.

3. Marston Morse, The calculus of variations in the large, Amer. Math. Soc. Colloquium Publications, vol. 18, New York, 1934.

4. Marston Morse, and Walter Leighton, Singular quadratic functionals, Trans. Amer. Math. Soc. vol. 40 (1936) pp. 252-286.

WASHINGTON UNIVERSITY AND

The Institute of Advanced Study 